\title{
Anesthetic management of a patient with obstructive prosthetic aortic valve dysfunction
}

\section{-a case report-}

\author{
Bo Ra Lee, Jeong-Rim Lee, and Min Soo Kim \\ Department of Anesthesiology and Pain Medicine, Anesthesia and Pain Research Institute, Yonsei University College of Medicine, \\ Seoul, Korea
}

We present a 55-year-old female patient who underwent burr-hole drainage due to chronic subdural hematoma, with obstructive prosthetic aortic valve dysfunction. Anesthetic management of a patient with severe obstructive prosthetic aortic valve dysfunction can be challenging. Similar considerations should be given to patients with aortic stenosis with an additional emphasis on thrombotic complication due to discontinuation of anticoagulation, which may further jeopardize the valve dysfunction. This case emphasizes the importance of a comprehensive understanding of the etiology and hemodynamic consequences of obstructive prosthetic valve dysfunction and the adequacy of anticoagulation for patients undergoing noncardiac surgery even after a successful valve replacement. (Korean J Anesthesiol 2014; 66: 160-163)

Key Words: Aortic valve stenosis, Echocardiography, Heart valve prosthesis, Thrombosis.

Even after a successful heart valve replacement without prosthetic valve malfunction, obstructive prosthetic valvular dysfunction (PVD) may occur due to prosthesis-patient mismatch (PPM), pannus formation, and thrombus formation [1]. Considering the relatively narrow anatomic feature of the left ventricular (LV) outflow tract, the aortic valve is more prone to develop PPM after valve replacement [2]. In patients who underwent aortic valve replacement due to severe stenosis, the persistence of an increased pressure gradient across the aortic valve would counteract the favorable reverse remodeling of concentric LV hypertrophy, which is well known to be associated with an adverse outcome [3]. Furthermore, in the case of mechanical heart valves, discontinuation of anticoagulation for other medicosurgical conditions may further aggravate the pressure imposed on the LV due to thrombus formation [4]. We herein report a case of a patient with obstructive PVD after mechanical aortic valve replacement for severe aortic stenosis, requiring burr-hole drainage for a subdural hematoma.

\section{Case Report}

A 55-year-old woman (height $153 \mathrm{~cm}$, weight $59.5 \mathrm{~kg}$ ) was scheduled for burr-hole drainage due to a subdural hematoma. Previous medical history revealed rheumatic involvement of

Received: July 23, 2012. Revised: 1st, September 15, 2012; 2nd, October 26, 2012; 3rd, December 25, 2012. Accepted: February $4,2013$.

Corresponding author: Min Soo Kim, M.D., Department of Anesthesiology and Pain Medicine, Anesthesia and Pain Research Institute, Yonsei University College of Medicine, 50, Yonsei-ro, Seodaemun-gu, Seoul 120-752, Korea. Tel: 82-2-2227-3561, Fax: 82-2-2227-7897, E-mail: kmsviola@yuhs.ac

(c) This is an open-access article distributed under the terms of the Creative Commons Attribution Non-Commercial License (http:// creativecommons.org/licenses/by-nc/3.0/), which permits unrestricted non-commercial use, distribution, and reproduction in any medium, provided the original work is properly cited. 
heart valves resulting in moderate mitral stenosis and severe aortic stenosis with a preserved LV ejection fraction (EF) of $83 \%$. Accordingly, the patient had undergone a double heart valve replacement (DVR) surgery 6 years ago with mechanical valves (Sorin $^{\circledR}$, Sorin group, Milano, Italy) \#18 and \#25 for the aortic and mitral valve, respectively. Echocardiography performed immediately after DVR revealed a LVEF of $60 \%$ and significant reductions in the peak and mean systolic pressure gradient (PSPG/MSPG) across the prosthetic valves. In detail, the PSPG/ MSPG across the aortic valve was reduced from $122 / 74 \mathrm{mmHg}$ to $29 / 12 \mathrm{mmHg}$. Follow-up echocardiography performed 2 years after DVR revealed an increased pressure gradient across the prosthetic aortic valve (PSPG/MSPG $=91 / 57 \mathrm{mmHg}$ ) due to PPM and possibly from pannus formation, with good motion of the prosthetic mitral valve and normal LVEF. Echocardiography performed a year ago revealed similar findings with persistence of the pressure gradient across the aortic valve (PSPG/MSPG = 98/50 mmHg) (Fig. 1). While the patient had been asymptomatic regarding obstructive PVD, her treatment plan was close medical observation.

Warfarin $7.5 \mathrm{mg}$ and olmesartan $10 \mathrm{mg}$ was given orally per day after DVR, and warfarin was discontinued as soon as the diagnosis of a subdural hematoma was confirmed. The patient's electrocardiography (ECG) showed a sinus rhythm with firstdegree AV block and other preoperative laboratory studies were all within the normal limits. As the patient was free of cardiac symptoms, an additional echocardiography was not performed before the burr-hole drainage.

We planned to perform general anesthesia under the assumption that her hemodynamic status was similar to a patient with severe, asymptomatic aortic stenosis. Upon arriving at the operating room, the patient was alert and standard monitoring devices were applied including 3-lead ECG, noninvasive blood

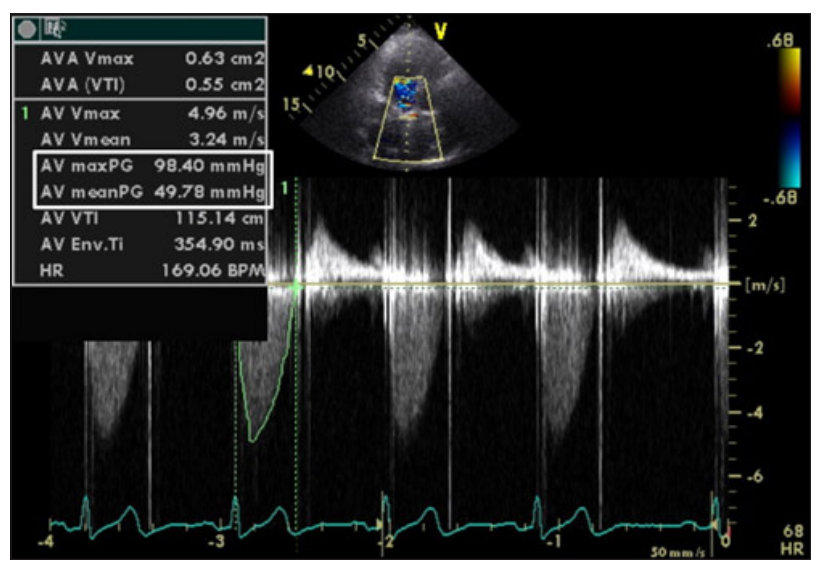

Fig. 1. Transthoracic echocardiography conducted a year ago revealed an increased pressure gradient through the prosthetic aortic valve (peak/ mean pressure $=98 / 50 \mathrm{mmHg}$ ). pressure and pulse-oximetry. Initially assessed vital signs were as follows: arterial blood pressure of 173/72 $\mathrm{mmHg}$, pulse rate of 63 beats/min, and peripheral oxygen saturation (SpO2) of $100 \%$ in room air. Glycopyrrolate $0.1 \mathrm{mg}$ was given as premedication and radial artery cannulation under local anesthesia was performed for continuous monitoring of arterial blood pressure before anesthetic induction. Anesthesia was induced via the intravenous route using midazolam $3.5 \mathrm{mg}$, thiopental sodium $50 \mathrm{mg}$, and a continuous infusion of remifentanil using a target-controlled infusion device (Orchestra ${ }^{\circledR}$ infusion pump system, Fresenius vial, Brenzins, France). The effect site concentration of remifentanil was gradually increased while paying attention to the patient's vital signs. After loss of consciousness, mask ventilation was maintained with $1.5 \%$ of sevoflurane in $100 \%$ oxygen, and $30 \mathrm{mg}$ of rocuronium bromide was injected intravenously. After the effect site concentration of remifentanil reached $4.0 \mathrm{ng} / \mathrm{ml}$, the trachea was intubated and the lungs were mechanically ventilated with $60 \%$ oxygen with air. Mechanical ventilation was performed to achieve an end-tidal carbon dioxide level between 35 and 40 $\mathrm{mmHg}$. Her heart rate remained unchanged while her blood pressure was maintained between 130/70 $\mathrm{mmHg}$ and 140/80 $\mathrm{mmHg}$ during the period of anesthetic induction and tracheal intubation. For anesthesia maintenance, sevoflurane was carefully titrated to maintain a bispectral index score between 40 and 60. The effect site concentration of remifentanil was adjusted to maintain the intraoperative blood pressure and heart rate within $20 \%$ of the preoperative values. Major hemodynamic goals during the surgery were to maintain the systolic blood pressure between 130-170 $\mathrm{mmHg}$, diastolic blood pressure between 60-80 mmHg, and pulse rate between $55-70$ beats/min. Upon completion of the surgery, sevoflurane was discontinued, and the neuromuscular blockade was antagonized with $0.2 \mathrm{mg}$ of glycopyrrolate and $1 \mathrm{mg}$ of neostigmine. After confirmation of regaining adequate consciousness and spontaneous respiration, the patient was transferred to the post anesthesia recovery unit. The duration of the surgery and anesthesia was 30 and $60 \mathrm{~min}$, respectively, and $200 \mathrm{ml}$ of colloid solution and $300 \mathrm{ml}$ of crystalloid solution were given. There were no remarkable events in the operating room and in the post anesthesia recovery unit.

Two days after the operation, transthoracic echocardiography was performed, and it still showed an increased aortic valvular pressure gradient (PSPG/MSPG $=100 / 55 \mathrm{mmHg}$ ), an aortic valve area (AVA) of $0.88 \mathrm{~cm}^{2}$ and an indexed effective orifice area (EOA) of $0.56 \mathrm{~cm}^{2}$. While warfarin could not be restarted immediately, a follow-up transesophageal echocardiography was performed on the tenth day after operation, which revealed a hyperechogenic fixed structure at right coronary cusp side, which seemed to be suspicious of a pannus formation or organized thrombus (Fig. 2). In addition, good motion of the prosthetic aortic valve with flow acceleration, partial motion limita- 

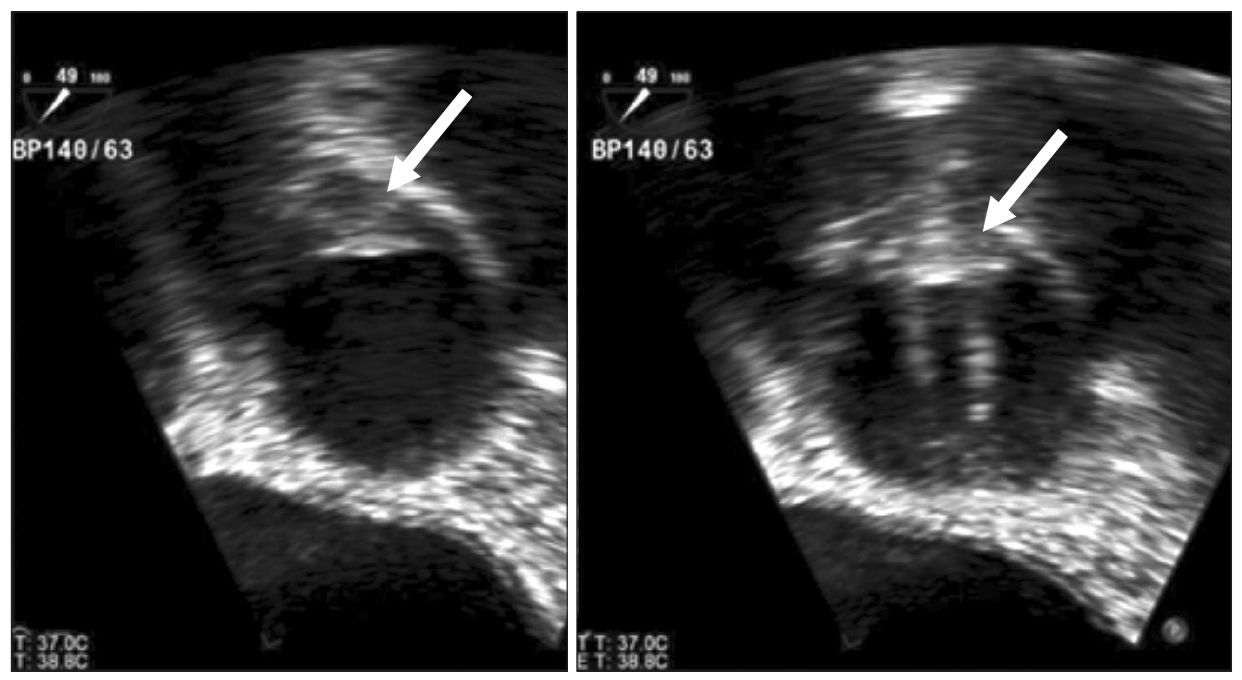

Fig. 2. Transesophageal echocardiography on the tenth day after the operation revealed a hyperechogenic fixed structure at the right coronary cusp side, which seemed to be suspicious of a pannus formation or organized thrombus.

tion of the anterolateral leaflets of the prosthetic mitral valve, and moderate mitral stenosis (mid-diastolic pressure gradient $=10 \mathrm{mmHg}$ ) probably due to thrombus formation were also observed. Despite these findings, warfarin could not be started until 2 weeks after the surgery to prevent fatal hemorrhagic complications. Heart computer tomography on the eighteenth day after the operation showed no significant coronary artery disease, no prosthetic mitral valve dysfunction, and a wellfunctioning prosthetic aortic valve. Follow-up echocardiography performed upon discharge 3 weeks after the operation revealed a slightly decreased pressure gradient across the aortic valve (PSPG/MSPG $=89 / 50 \mathrm{mmHg}$ ) and a well-functioning prosthetic mitral valve with loss of the previously observed pressure gradient with a LVEF of $73 \%$. The patient was discharged without any complications.

\section{Discussion}

Anesthetic management of a patient with obstructive PVD can be challenging. In particular, the obstructive PVD at the aortic site has hemodynamic properties similar to aortic stenosis such as obstruction of LV ejection, elevated transvalvular pressure gradient and concentric LV hypertrophy $[5,6]$. The continuous and fixed high-pressure imposed on the LV can interfere with the favorable reverse remodeling of the LV hypertrophy normally observed after successful valve replacement [7]. Limited regression of LV hypertrophy is associated with higher long-term mortality and increased risk of complications such as stroke, sudden death and recurrent cardiac symptoms [3]. Various causes have been identified for the development of obstructive PVD including PPM, pannus and/or thrombus formation [1].

PPM was described as elevation of the transvalvular pressure gradient generated by the smaller effective area of the inserted prosthetic valve compared to that of the native human valve [8]. The parameter for measuring the severity of PPM is the EOA of the valve which is the EOA of the prosthesis divided by the patient's body surface area (BSA, indexed EOA = EOA/BSA) [2]. Pannus formation is an overgrowth of fibrous tissue composed of elastic tissue and collagen with chronic inflammatory cells, endothelial cells and myofibroblasts [1]. Although the exact etiology is unknown, a variety of factors are involved in its formation. Recent reports showed that prosthetic valve dysfunction with pannus was related to a significant elevation in the level of transforming growth factor beta 1 , which plays an important role in the growth and differentiation of cells and matrix formation [5]. Thrombus formation is the most common problem with an approximate incidence of $4 \%$ per patient-year and anticoagulation reduces the risk to $1 \%$ per patient-year [9]. Although challenging, a differential diagnosis of thrombus from pannus formation in obstructive PVD is very important in terms of the use of thrombolytic therapy [10]. Anticoagulation status, duration of symptom, and ultrasound intensity of the lesion causing the obstructive PVD can help differentiate thrombus from pannus formation [10]. Generally, the treatment in asymptomatic patients with PPM or pannus is regular follow-up without reoperation despite the high transvalvular pressure gradient. Thrombus can be resolved by adequate anticoagulation and thrombolysis [10]. However, thrombolytic therapy cannot be applied in patients at a risk of hemorrhagic complications as in this case.

In the current case, a high-pressure gradient across the prosthetic aortic valve corresponding to severe aortic stenosis due to PPM and/or pannus formation was observed in the preoperative echocardiography. Thus, anesthetic considerations for the noncardiac surgery of patients with severe aortic stenosis were applied for this case although this patient was free of cardiac symptoms. Notwithstanding the high mortality in patients 
with severe aortic stenosis undergoing noncardiac surgery (up to $15 \%$ ) [11], this patient had an unremarkable perioperative course probably due to the following reasons. Besides being free of cardiac symptoms, another reason for the observed favorable prognosis was that she did not have severe concentric LV hypertrophy ( $\mathrm{LV}$ mass index $=101 \mathrm{~g} / \mathrm{m}^{2}$ ) even in the presence of severe obstructive PVD [12]. Actually, patients with concentric LV hypertrophy suffer from congestive heart failure due to diastolic dysfunction despite preserved systolic function. No cardiac symptoms and no severe LV hypertrophy in the preoperative echocardiography may serve as good prognostic factors in patients with obstructive PVD undergoing noncardiac surgery.

Although sharing a similar pathophysiology, an additional problem that needs to be addressed in patients with obstructive PVD in contrast to patients with aortic stenosis is discontinuation of anticoagulation in the presence of other life-threatening medico-surgical co-morbidities. Adequate anticoagulation is essential to prevent thrombus formation in patients with mechanical prosthetic valves. Usually, withholding of anticoagulation in patients with prosthetic heart valves and intracranial hemorrhage for less than 7 days was related to low thromboembolic incidence and there was no clinical evidence of prosthetic heart valve dysfunction when anticoagulation was stopped for less than 14 days [4]. In this case, however, anticoagulation was withheld for 4 weeks ( 2 weeks prior to the surgery upon diagnosis confirmation and 2 weeks after the surgery) jeopardizing the integrity of the prosthetic valves. Indeed, a newly developed increased pressure gradient was observed across the mitral valve with some motion limitation indicating the presence of thrombus. Fortunately, the pressure gradient and dysfunction of the mitral valve were resolved after anticoagulation in the current case without affecting the already increased pressure gradient across the aortic valve. By considering the risk/benefit ratio of anticoagulation therapy in such a patient population, close follow-up and communication with the surgeon and cardiologist seem to be an absolute necessity to prevent any mishaps jeopardizing the outcome of patients.

In conclusion, the presence of obstructive prosthetic valve dysfunction in a noncardiac surgical setting can be a serious challenge to anesthesiologists. When present at the aortic site, similar considerations should be given as in patients with aortic stenosis with an additional emphasis on thrombotic complications due to discontinuation of anticoagulation. This case emphasizes the importance of a comprehensive understanding of the etiology and hemodynamic consequences of obstructive PVD and the adequacy of anticoagulation for patients undergoing noncardiac surgery even after a successful valve replacement.

\section{References}

1. Toker ME, Eren E, Balkanay M, Kirali K, Yanartaş M, Calişkan A, et al. Multivariate analysis for operative mortality in obstructive prosthetic valve dysfunction due to pannus and thrombus formation. Int Heart J 2006; 47: 237-45.

2. Apostolakis E, Baikoussis NG, Papakonstantinou NA, Goudevenos J. Patient-prosthesis mismatch and strategies to prevent it during aortic valve replacement. Hellenic J Cardiol 2011; 52: 41-51.

3. Perez de Arenaza D, Lees B, Flather M, Nugara F, Husebye T, Jasinski M, et al. Randomized comparison of stentless versus stented valves for aortic stenosis: effects on left ventricular mass. Circulation 2005; 112: 2696-702.

4. Krittalak K, Sawanyawisuth K, Tiamkao S. Safety of withholding anticoagulation in patients with mechanical prosthetic valves and intracranial haemorrhage. Intern Med J 2011; 41: 750-4.

5. Darwazah AK. Recurrent pannus formation causing prosthetic aortic valve dysfunction: is excision without valve re-replacement applicable? J Cardiothorac Surg 2012; 7: 62.

6. Nathaniel S, Saligram S, Innasimuthu AL. Aortic stenosis: An update. World J Cardiol 2010; 2: 135-9.

7. Del Rizzo DF, Abdoh A, Cartier P, Doty D, Westaby S. Factors affecting left ventricular mass regression after aortic valve replacement with stentless valves. Semin Thorac Cardiovasc Surg 1999; 11(4 Suppl 1): 114-20.

8. Rahimtoola SH. The problem of valve prosthesis-patient mismatch. Circulation 1978; 58: 20-4.

9. Cannegieter SC, Rosendaal FR, Briët E. Thromboembolic and bleeding complications in patients with mechanical heart valve prostheses. Circulation 1994; 89: 635-41.

10. Barbetseas J, Nagueh SF, Pitsavos C, Toutouzas PK, Quinones MA, Zoghbi WA. Differentiating thrombus from pannus formation in obstructed mechanical prosthetic valves: an evaluation of clinical, transthoracic and transesophageal echocardiographic parameters. J Am Coll Cardiol 1998; 32: 1410-7.

11. Mittnacht AJ, Fanshawe M, Konstadt S. Anesthetic considerations in the patient with valvular heart disease undergoing noncardiac surgery. Semin Cardiothorac Vasc Anesth 2008; 12: 33-59.

12. Ganau A, Devereux RB, Roman MJ, de Simone G, Pickering TG, Saba PS, et al. Patterns of left ventricular hypertrophy and geometric remodeling in essential hypertension. J Am Coll Cardiol 1992; 19: 1550-8. 